\title{
A new chapter...
}

\author{
Bert Gordijn $\cdot$ Henk ten Have
}

Published online: 11 March 2010

(C) Springer Science+Business Media B.V. 2010

This is a crucial time in the development of "Medicine, Health Care and Philosophy". First, there is an important change within the editorial board. Wim Dekkers has left and Henk ten Have has rejoined the board as editor-inchief. Against this backdrop, Henk and I would like to take this opportunity to express our thanks to Wim for his service for the journal over the past 12 years, first as managing editor and subsequently as editor-in-chief. Second, Wim is not leaving the journal in a bad state. In actual fact it is stronger than it has ever been before: it has been accepted by ISI, Thomson Reuters' abstracting and indexing service. As a result the journal will now be abstracted and indexed in: the 'Social Sciences Citation Index', the 'Arts and Humanities Citation Index', the 'Current Contents Social and Behavioural Sciences' as well as the 'Current Contents Arts \& Humanities'. We trust this positive development will encourage you to keep on submitting first-rate manuscripts to our unique journal.

This current issue of "Medicine, Health Care and Philosophy" focuses on a series of questions fairly close to clinical practice. The first two papers consider the way in which medical professionals engage with ethical dimensions of their daily work. In "Clinical essentialising: a qualitative study of doctors' medical and moral practice", Kari Agledahl, Reidun Førde and Åge Wifstad observe a very similar pattern across different medical specialities, in which physicians transform patients' diverse concerns into

B. Gordijn ( $\square)$

Institute of Ethics, Henry Grattan Building, Dublin City

University, Dublin 9, Ireland

e-mail: bert.gordijn@dcu.ie

H. ten Have

UNESCO, Paris, France specific medical questions, a process dubbed 'essentialising'. In so doing doctors tend to focus on benefiting patients' physical and mental functions. Their small exploratory study suggests that essentialising should not be seen as a shortcoming of individual doctors. Rather it is the product of their systematic clinical approach and an integral part of clinical practice. Next, Joseph Kaufert et al. argue that the type of care patients receive is a function of the way in which medical professionals tackle ethical issues. This becomes particularly important when dealing with end-of-life situations of patients with disabilities. Against this backdrop, the authors explore new ways of teaching disability perspectives on end-of-life ethics to first year medical students departing from traditional teaching in this area. Moreover, they consider how these novel teaching methods are evaluated by a range of stakeholders.

The next two articles analyse issues surrounding diagnosis. In "Too much of a good thing is wonderful?", Bjørn Hofmann examines unwarranted examinations and diagnostic futility in diagnostic radiology. The author argues that the question of whether radiological examinations should be regarded as excessive hinges on the criterion for diagnostic futility. However, such a criterion is still lacking in the literature. Thus Hofmann develops a new definition of diagnostic futility. Next, Marianne Boenink and Simone van der Burg focus on the process of informed decision making about predictive DNA tests. Since the latter may have considerable impact on the tested individual's life the decision whether to undergo a test is regarded as a matter up to the individual. Therefore, the client should be free to choose, at least in liberal societies. This means, of course, that there should be no outside coercion with the client's personal decisions (i.e. negative freedom). In addition however, the authors argue that the client should be actively enabled to make good choices (positive freedom). 
Thus the authors consider how individuals and families might be supported in their deliberations, without interfering with their actual decisions.

Similarly, in the next paper, Mare Knibbe and Marian Verkerk argue that respect for autonomy involves more than merely lending a hand to individual self determination through standard prerequisites of informed consent. The authors focus on donor risk communication in families considering living liver donation to a child, where responsible decision making is embedded within family processes. As a consequence, proper risk communication is difficult to grasp in the terms of the dyadic physician/donor relation. Thus, good donor risk communication must be conceived within the broader social context of the changes that a family undergoes in the process of decision making.
Accordingly, transplant professionals have to enlarge their traditional role and responsibility for risk communication.

In the penultimate paper of this issue, Patrick Stefan Kermit examines the two different post-operative trajectories for children with cochlear implants, one with sign language and spoken language simultaneously and the other with speech only. The author advances the precautionary principle as an instrument that might help choose between these two options. The final article, by Carla Truyers et al., focuses on two new biobanks in Belgium. Up to now, there is a lack of regulatory frameworks tackling the storage of human tissue in Belgium, or so the authors argue. Hence, they analyse various ethical and legal aspects and present a set of recommendations with regard to issues of consent, privacy, harms and ethics boards. 